University of Nebraska - Lincoln

DigitalCommons@University of Nebraska - Lincoln

3-9-1992

\title{
Backward Peak in the Electron Spectrum from Collisions of 70-keV Protons with a Target from a Hydrogen-Atom Source
}

\author{
M. Eugene Rudd \\ M. W. Gealy \\ University of Nebraska - Lincoln \\ G. W. Kerby, III \\ University of Nebraska - Lincoln \\ Ying-Yuan Hsu \\ University of Nebraska - Lincoln
}

University of Nebraska - Lincoln, erudd@unl.edu

Follow this and additional works at: https://digitalcommons.unl.edu/physicsrudd

Part of the Physics Commons

Rudd, M. Eugene; Gealy, M. W.; Kerby, III, G. W.; and Hsu, Ying-Yuan, "Backward Peak in the Electron Spectrum from Collisions of 70-keV Protons with a Target from a Hydrogen-Atom Source" (1992). M. Eugene Rudd Publications. 46.

https://digitalcommons.unl.edu/physicsrudd/46

This Article is brought to you for free and open access by the Research Papers in Physics and Astronomy at DigitalCommons@University of Nebraska - Lincoln. It has been accepted for inclusion in M. Eugene Rudd Publications by an authorized administrator of DigitalCommons@University of Nebraska - Lincoln. 


\title{
Backward Peak in the Electron Spectrum from Collisions of 70-keV Protons with a Target from a Hydrogen-Atom Source
}

\author{
M. E. Rudd, M. W. Gealy, (a) G. W. Kerby, III, and Ying-Yuan Hsu \\ Department of Physics and Astronomy, University of Nebraska, Lincoln, Nebraska 68588-0111
}

(Received 30 September 1991)

\begin{abstract}
A large, broad peak has been found in the electron energy spectrum from 30 to $100-\mathrm{keV}^{+}+\mathrm{H}^{\text {col- }}$ lisions. The peak, which is centered at $31 \mathrm{eV}$, appears only in the backward directions. It is suggested that while the peak is unlikely to come from collisions with atoms in the ground or metastable states, it could arise from collisions with atoms in highly excited states or from autoionizing states of the residual $\mathrm{H}_{2}$ in the target.
\end{abstract}

PACS numbers: $34.50 . \mathrm{Fa}, 34.50 . \mathrm{Gb}$

Cross sections for collisional ionization as a function of the energy and direction of the ejected electron have been measured for many target atoms and molecules and for several incident particles. Because of experimental problems, however, atomic hydrogen has not been used as a target for this kind of measurement until very recently. Shyn [1] has made such measurements for electron impact and we have undertaken to do the same for proton and other ion impact. During the course of this work we discovered a large unexpected peak in the electron spectrum from $\mathrm{H}^{+}+\mathrm{H}$ collisions in the backward hemisphere not seen in the electron impact work. This peak, which is centered at about $31 \mathrm{eV}$, is the subject of this Letter. Although the peak accounts for about $35 \%$ of the integrated cross section at $160^{\circ}$, it falls off rapidly with decreasing angle and provides only about $2 \%$ of the total electron ejection cross section at $70 \mathrm{keV}$.

The apparatus and experimental method will be described more fully in a forthcoming publication but briefly, a magnetically analyzed beam of protons goes through a well-pumped beam line $\left(P \sim 4 \times 10^{-7}\right.$ Torr $)$ into a stainless-steel chamber. After passing through the target gas at the collision center it is collected by a biased Faraday cup and integrated. Electrons from the collision center enter a $180^{\circ}$ hemispherical electrostatic analyzer with a resolution of $5 \%$. The analyzer is rotatable from $15^{\circ}$ to $165^{\circ}$ relative to the ion beam direction. A channeltron detects the analyzed electrons. A single external coil combined with magnetic shielding inside the chamber suffice to reduce the magnetic field in the chamber to a few milligauss.

Atomic hydrogen is obtained by dissociating $\mathrm{H}_{2}$ in a radio-frequency (rf) source designed by Slevin and Stirling [2] and built in England [3]. Hydrogen gas is admitted through a palladium finger to a Pyrex glass tube which is water cooled. Radio-frequency power at 35 $\mathrm{MHz}$ is fed to a high- $Q$ cavity to excite the discharge. The coil is well shielded to reduce $\mathrm{rf}$ noise from escaping. The atomic hydrogen effuses from a $1-\mathrm{mm}$ capillary which has a kink to block the ultraviolet light emitted by the source. After crossing the proton beam, the atomic beam enters the inlet of a diffusion pump with a pumping speed for hydrogen of $1700 \mathrm{~L} / \mathrm{s}$. The chamber pressure typically rises only to about $8 \times 10^{-7}$ Torr when hydrogen is flowing from the source.

We determine the dissociation fraction for each run by reversing the polarities of the analyzer and detector and comparing the numbers of $9-\mathrm{eV}$ positive ions produced by the proton beam in the $\mathrm{H} / \mathrm{H}_{2}$ mixture with the $\mathrm{rf}$ on and off. Ions of that energy are produced by dissociation in $\mathrm{H}^{+}+\mathrm{H}_{2}$ collisions [4], but not in $\mathrm{H}^{+}+\mathrm{H}$ collisions. With source inlet pressures of $0.03-0.1 \mathrm{mB}$, dissociation fractions of $90 \%$ and higher are usually obtained.

Figure 1 shows a part of the electron spectrum observed at $160^{\circ}$ for $70-\mathrm{keV}$ protons with the rf off $(100 \%$ $\mathrm{H}_{2}$ ) and on $\left(4 \% \mathrm{H}_{2}\right.$, the remaining $96 \%$ presumably $\left.\mathrm{H}\right)$. Nothing in the apparatus was changed between the two runs except for the switching of the $\mathrm{rf}$. Therefore the peak does not appear to be apparatus related. In an attempt to understand the origin of the peak we performed several tests. To eliminate the possibility that the rf sig-

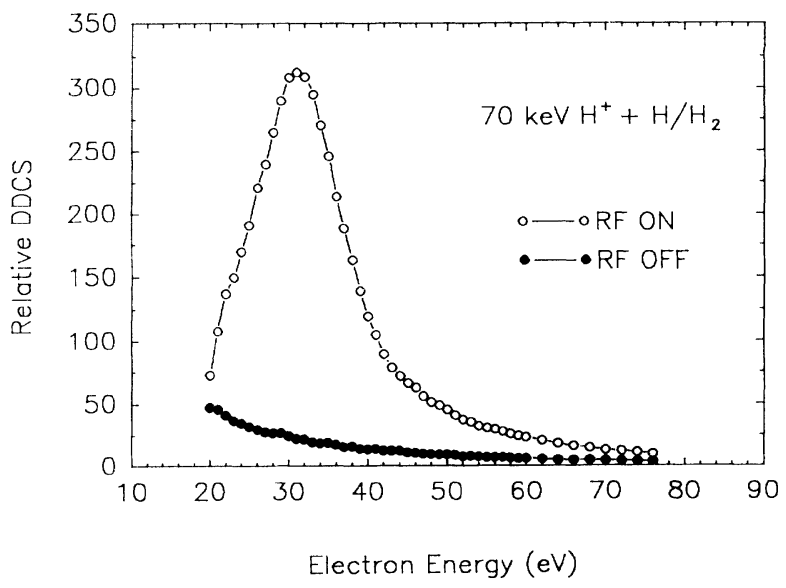

FIG. 1. Spectrum of electrons ejected at $160^{\circ}$ from $70-\mathrm{keV}$ proton impact on the target gas from the Slevin hydrogen atom source. Open circles, the $\mathrm{rf}$ excitation was on and the measured dissociation fraction was $93 \%$; solid circles, the $\mathrm{r}$ f excitation was off, making the target pure $\mathrm{H}_{2}$. 
nal itself was the cause, we constructed a system that switches the rf on and off at a rate of $50 \mathrm{~Hz}$ with a $50 \%$ duty cycle. Electrons were counted only during the halfcycle when the rf was off. Auxiliary measurements have shown that after deactivating the rf excitation, the atomic hydrogen from the source decays very slowly (the time constant is approximately $1 \mathrm{~min}$ ) so there is no appreciable decay during a switching cycle. The data taken in this way are virtually identical with the measurements taken with the rf on continuously, showing that the rf has little or no direct effect on the detector or the counting system. Furthermore, turning off the proton beam reduces the count rate to a negligible background rate of a few per minute even when the rf is on. This background is only slightly higher with the rf and shows no dependence on the energy setting of the analyzer. We also substituted nitrogen for hydrogen in the source and found no peak with or without the rf and with or without the beam.

Electron spectra taken at proton energies of $30,50,70$, and $100 \mathrm{keV}$ showed no difference in the electron energy at which the peak appears. However, the height of the peak varies, reaching a maximum at about $70-100 \mathrm{keV}$ and decreasing to a small value at $30 \mathrm{keV}$. Likewise, the position of the peak does not vary with the angle of emission, but the height decreases as the angle is decreased below $160^{\circ}$, disappearing at $90^{\circ}$. To see if the angular dependence is due to an asymmetry in the $\mathrm{H}$-atom source, we rotated the top of the chamber (which carries the source) by $165^{\circ}$ but found no change in the angular distribution relative to the ion beam. In another test, the peak was still present, although smaller, when a $\mathrm{He}^{+}$incident beam was used in place of $\mathrm{H}^{+}$. In order to determine whether charged particles such as electrons or $\mathrm{H}^{-}$ were coming from the $\mathrm{H}$-atom source, a normally grounded internal electrode near the tip was connected to a source of potential which was varied up to $-40 \mathrm{~V}$ without effect on the peak.

Two lines of evidence indicate that the peak is due to an unstable component of the beam from the $\mathrm{H}$-atom source. When the density of the target was varied by changing the pressure of the hydrogen at the inlet to the source, it was found that the ratio of the peak signal to the target density decreased with increasing source pressure. In fact, at a high enough source pressure $(0.2 \mathrm{mB})$, the peak disappears. This seems to indicate that the component of the target responsible for the peak is destroyed by gas collisions. In a second test, the distance between the tip of the source and the proton beam was varied. It was found that the signal at the $31-\mathrm{eV}$ peak energy falls off much faster with distance than signals at energies on either side of the peak, indicating an in-flight decay of the particles. Assuming that the unstable particles from the source are hydrogen atoms moving with thermal velocities, their decay rate with distance is consistent with a lifetime of the order of $10^{-7} \mathrm{~s}$. This rules out the meta- stable $2 s$ state which has a much longer lifetime in lowfield environments. However, many of the higher excited states of the hydrogen atom have lifetimes of about $10^{-7}$ s [5].

While the peak is still unexplained, we can speculate on its origin. One possibility is that collisions with hydrogen atoms in highly excited states could produce electrons in the backward directions. Schultz et al. [6] have recently made extensive classical-trajectory Monte Carlo (CTMC) calculations of electron ejection cross sections for $\mathrm{H}^{+}+\mathrm{H}(1 s, 2 s)$ collisions. While their calculated values at $70 \mathrm{keV}$ for the ground-state target are in excellent agreement with our measured cross sections in the forward direction, they show no evidence of a peak in the backward direction. However, their results for the $2 s$ target atoms show a backward enhancement, but at electron energies much lower than $31 \mathrm{eV}$. If similar calculations for atoms in higher excited states showed such a peak at the measured energy, this would indicate a hitherto unsuspected mechanism for ejection of electrons in a direction opposite to that of the beam.

A second possibility is that in addition to atomic hydrogen the Slevin source may produce hydrogen molecules in doubly excited states from which autoionization could be induced by the field of a passing proton. McGowan et al. [7] attributed some aspects of their measurements of electron impact dissociation of $\mathrm{H}_{2}$ to autoionizing states with lifetimes of $10^{-7}$ to $10^{-6} \mathrm{~s}$. There are several such states with energies of the right value to account for the energy of the peak [8]. Alternatively, the molecules from the source may be in a lower excited state from which the proton collisions could raise them to higher autoionizing states. If confirmed, these results may suggest mechanisms for exciting and studying such states. None of the proposed mechanisms, however, accounts for the unusual angular distribution.

Discussions have been held with many people concerning the origin of the backward peak. The authors especially wish to thank D. H. Jaecks, J. A. R. Samson, L. Toburen, and J. Macek for helpful ideas and D. R. Schultz and R. E. Olson for communicating the results of their CTMC calculations in advance of publication. This paper is based on work supported by the National Science Foundation under Grants No. PHY8701905 and No. PHY9020529.

(a) Present address: Concordia College, Moorhead, MN 56562.

[1] T. W. Shyn (private communication).

[2] J. Slevin and W. Stirling, Rev. Sci. Instrum. 52, 1780 (1981).

[3] Leisk Engineering (now VSW Technology, Ltd.), Albert 
Drive, Burgess Hill, West Sussex, RH15 9NX, England.

[4] See, e.g., R. M. Wood, A. K. Edwards, and M. F. Steuer, Phys. Rev. A 15, 1433 (1977).

[5] H. A. Bethe and E. E. Salpeter, Quantum Mechanics of One- and Two-Electron Atoms (Springer-Verlag, Berlin, 1957), p. 266.
[6] D. R. Schultz, R. E. Olson, C. O. Reinhold, M. W. Gealy, George W. Kerby, III, Ying-Yuan Hsu, and M. E. Rudd, J. Phys. B 24, L599 (1991).

[7] J. W. McGowan, M. A. Fineman, E. M. Clarke, and H. P. Hanson, Phys. Rev. 167, 52 (1968).

[8] T. E. Sharp, At. Data 2, 119 (1971). 